\title{
Micropipette-powered droplet based microfluidics
}

\author{
Krzysztof Langer, Nicolas Bremond, ${ }^{\text {a) }}$ Laurent Boitard, Jean Baudry, \\ and Jérôme Bibette \\ Laboratoire Colloïdes et Matériaux Divisés, CNRS UMR 8231, Chemistry Biology \& Innovation, \\ ESPCI Paris, PSL Research University, 10 rue Vauquelin, F-75005 Paris, France
}

(Received 27 April 2018; accepted 26 June 2018; published online 10 July 2018)

Droplet-based microfluidics, using water-in-oil emulsion droplets as micro-reactors, is becoming a widespread method for performing assays and especially in the cell biology field. Making a simple and highly portable system for creating emulsion droplets would help to continue the popularization of such a technique. Also, the ability to emulsify all the samples would strengthen this compartimenlization technique to handle samples with limited volume. Here, we propose a strategy of droplet formation that combines a classical flow-focusing microfluidic chip, which could be commercially available, with a standard laboratory adjustable micropipette. The micropipette is used as a negative pressure generator for controlling liquid flows. In that way, emulsification does neither require any electrical power supply nor a cumbersome device and functions with small liquid volumes. Droplet formation can be easily and safely performed in places with limited space, opening a wide range of applications especially in biological laboratory environments with higher level of safety regulations, i.e., BSL-3/4. Fortunately, the present methodology that involves small fluid volumes, and thus possible time dependent flow conditions, allows to minimize dead volume while keeping drops' size homogeneous. A physical characterization of droplet production and a model that describes the emulsion features, in terms of drop size and size distribution, are proposed for rationalizing the performances of the micropipette-powered emulsification process. Published by AIP Publishing. https://doi.org/10.1063/1.5037795

\section{INTRODUCTION}

The ability to perform compartmentalized reactions in emulsion droplets together with the development of microfluidics technology opened the way to a myriad of applications in chemistry and biology. ${ }^{1}$ Indeed, droplet-based microfluidics offers an unique possibility to precisely handle and control low volumes of fluid, as well as an ability to efficiently process multiple samples. Among others, a key advantage is the capability to study cells at the single-cell level ${ }^{2}$ or to precisely quantify target nucleic acids in a sample by polymerase chain reaction in droplets whose size homogeneity matters. ${ }^{3}$

Microfluidic chips designed for droplet generation can be fabricated using various methods ${ }^{4}$ (e.g., soft lithography or via hot embossing) and some of them can be directly purchased from microfluidic chip manufacturing companies at moderate costs. Then, one needs to control flow with either syringe pumps or pressure controllers. These devices allow to precisely control the liquid flow rates but are concomitant with dead volumes, mainly because of tubing connected to the microfluidic chips, and are not best suited for handling small sample volumes. Since some applications require to manipulate small and precious samples with a need to emulsify the whole sample, like, for example, when rare cells are tracked, other strategies are needed.

Alternative methods for driving liquid flows have been developed including syringevacuum microfluidics, ${ }^{5}$ a self-powered microfluidic mono dispersed droplet generator, ${ }^{6}$ as well

${ }^{a)}$ E-mail: Nicolas.Bremond@espci.fr 
as a finger-powered microfluidic system, ${ }^{7} 3 \mathrm{D}$ printed pumping $\operatorname{lid},{ }^{8}$ and a most recent method that uses a micropipette to push liquid to form droplets of a few tens of nano-liter droplets, i.e., a size of a few hundreds of $\mu \mathrm{m} .{ }^{9}$

It is also worth highlighting that nowadays most of the published results of experiments regarding droplet microfluidics were performed with non- or little pathogenic microorganisms. Due to the maturation of this scientific field and, in general, overall increased popularity of microfluidics, it is just a matter of time for droplet-based microfluidics to become commonly used with microorganisms that require laboratory facilities with a higher biosafety level (BSL 3 or BSL 4). According to World Health Organization's (WHO) Laboratory biosafety manual in a Biosafety Level 3 and 4 microbiological laboratory, any manipulation of all potentially infectious material must be conducted within a biological safety cabinet or other primary containment device. Hence, a syringe pump or any other equipment required to generate droplets together with microfluidic droplet generating chip should be placed inside a biological safety cabinet, not only taking precious space but also being exposed routinely to decontamination using often chlorine-releasing compounds. Those chemicals are fast-acting oxidants and are widely available broad spectrum chemical germicides. It also means that any expensive equipment decontaminated with chlorine-releasing compounds would be routinely exposed to highly alkaline solutions that can be corrosive to metal.

Here, we propose a rather easy and portable method for generating emulsion droplets. The idea is to use a micropipette for pumping the two immiscible liquids from pipette tips through a microfluidic chip composed of a flow-focusing geometry. The micropipette thus acts as a negative pressure generator. This strategy is naturally adapted to handle small volume while minimizing loss of sample. In the following, we first present the methodology and the corresponding emulsion features. Then, a more detailed characterization of the whole process is given along with a model that predicts the emulsion features, in terms of drop size and size distribution, and thus rationalize the performances of the micropipette-powered emulsification process.

\section{RESULTS AND DISCUSSION}

\section{A. Materials and methods}

There exist several processes for making emulsion droplets in microfluidics and the majority of them take advantage of the precise control of the confinement. ${ }^{10}$ Here, we used a flowfocusing geometry where droplets are formed at a cross-junction. ${ }^{11}$ The microfluidic chips were fabricated by using standard soft lithography techniques. ${ }^{12}$ The homemade microsystem are made of poly(dimethylsiloxane) (PDMS) (Sylgard 184, Dow Corning). The channel's height $h$ is $85 \mu \mathrm{m}$ and its width $w$ is equal to $65 \mu \mathrm{m}$ at the cross junction and to $150 \mu \mathrm{m}$ anywhere else. In order to maintain a good airtight connection between PDMS chip and a pipette tip, the inlets and outlets were punched with a $1 \mathrm{~mm}$-diameter biopsy punch (Harris Uni-Core, Electron Microscopy Sciences). In addition, the PDMS slab thickness should at least equal to $7 \mathrm{~mm}$. This lower limit is due the use of a $1 \mathrm{~mm}$ biopsy punch together with a micropipette's tip having a conical shape. Indeed, the connection is not airtight enough for thin PDMS slab and air bubbles might be introduced during the process. On the other hand, the biopsy punches are limited to $10 \mathrm{~mm}$ thick slab. Then, the PDMS slab was bonded onto a glass slide after surface activation using an oxygen plasma cleaner (FEMTO, Diener electronic). Following bonding, the surface properties of the microchannel's walls were modified with $1 \%$ solution of trichloro $(1 \mathrm{H}, 1 \mathrm{H}, 2 \mathrm{H}, 2 \mathrm{H}$-perfluorooctyl)silane (Aldrich) in HFE-7500 fluorocarbon oil (3M Novec 7500 Engineered Fluid). Silane was removed from microchannels using HFE-7500 fluorocarbon oil. Finally, all channels were dried and cleaned with compressed nitrogen. All solutions were filtered with $0.2 \mu \mathrm{m}$ filter to eliminate any particle contamination. We have tested microfluidic droplet generating chips from the company ChipShop (catalog number: 13-10070440-02) with 4 different geometries of flow focusing regions. Each chip is fabricated in a transparent polymer and has 8 individual droplet generators with four different heights and widths: a pair of $80 \times 80 \mu \mathrm{m}, 70 \times 70 \mu \mathrm{m}, 60 \times 60 \mu \mathrm{m}$, and $50 \times 50 \mu \mathrm{m}$, respectively. All microfluidic channels were modified using the same protocol as described for the PDMS chips, but it 
is also possible to order droplet generators with already modified hydrophobic surface. The droplet generator chips are equipped with a mini luer lock. Male Mini Luer fluid connectors (catalog umber: 09-0548-0333-09) are then required to connect the micropipette's tip with the microfluidic chip. In that case, the micropipette tip fits well with the chips' connectors and a tight connection is possible by slightly pushing the tip against the plastic connector.

The continuous phase is a fluorocarbon oil (HFE-7500) with the addition of a Krytox-PEGKrytox surfactant (RAN Biotechnologies, 008-FluoroSurfactant) at 2\% wt. The dispersed phase is an aqueous one. It is either pure water, a suspension of overnight colony of green fluorescent Escherichia coli cells in LB medium having a corresponding optical density of 0.05 , as well as $0.16 \mu \mathrm{M}$ solution of green quantum dots (CdSSe/ZnS with carboxylic acid, Ocean NanoTech). The different phases are manipulated with a standard adjustable micro pipette (Eppendorf Research plus, 2-200 $\mu \mathrm{l}$ ).

A homemade 2D chamber, made with bonded two glass slides and Nanoports for injecting fluids ${ }^{13}$ was used for assessing droplet size distribution. After having collecting emulsion droplets, the gap size between glass slides is such that the compact emulsion form a single layer of droplets. The droplet formation dynamics has been captured with a high speed camera (Fastcam-X 1024, Photron) mounted on an inverted microscope (DMI 3000B, Leica). Droplet features were analysed using image processing programs developed with Matlab.

\section{B. Making emulsion droplets with a micropipette}

The overall workflow of emulsification assisted by micropipette is illustrated in Fig. 1 (see also the movie in the supplementary material). First, the microsystem is filled with the continuous phase by injecting the oil, containing surfactants, through the oil inlet by using a micropipette in a pushing mode [Fig. 1(a)]. When the oil flows out of the two other ports, the micropipette is disconnected from the tip, while the tip is maintained plunged into PDMS. The chemical modification of the channels' surface and the addition of surfactant prevent any trapped bubbles inside the microsystem. Then, a micropipette tip filled with the phase to be dispersed is inserted into the aqueous phase inlet [Fig. 1(b)]. The micropipette's tip used as a collection reservoir and the micropipette itself can then be connected. After having set the sampled volume, the micropipette plunger is pressed to the maximum. In that way, an additional volume
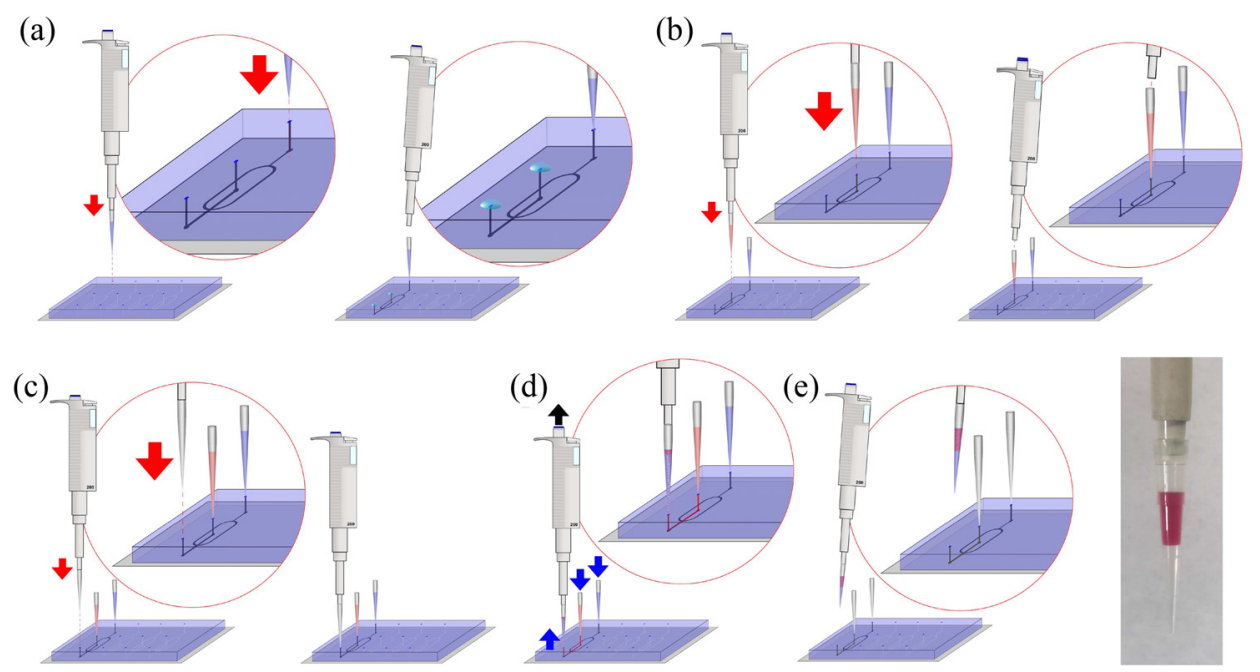

FIG. 1. Preparation steps for micropipette based droplet generation. (a) The chip is first filled with continuous phase (HFE7500 oil enriched with surfactant). Surface modification with a perfluoro-silane avoids to trap bubbles. The pipette tip containing oil is left connected to the chip. (b) A pipette tip filled with the phase to be dispersed is then connected to the chip. (c) An empty pipette tip, which is attached to the pipette with its plunger pressed at maximum, is finally connected to the chip. (d) Releasing the pipette plunger immediately starts droplet generation as the corresponding negative pressure induces the flow of both liquids through a flow-focusing device. (e) The emulsion droplets are collected into the pipette tip attached to the pipette that can be disconnected from the chip at any moment. 
of about $50 \mu \mathrm{l}$ is added to the set volume. The plunger is then released after a tight connection with the chip is established [Fig. 1(c)]. Releasing the pipette plunger immediately triggers the suction of the liquids from the two other tips and thus droplet generation when the liquids meet at the cross junction [Fig. 1(d)]. We note that a sudden release of the micropipette plunger may lead to initial heterogeneous droplet size. This can be prevented by accompanying the upward motion of the plunger with the thumb such that it takes a few seconds to reach its equilibrium position (see movie). The micropipette is thus used as a low pressure generator for inducing liquid flow, similarly to the syringe-based strategy previously proposed ${ }^{5}$ or more recently with the help of a pressure controller. ${ }^{14}$ Here, the sampled volume, and thus the pressure, is precisely controlled with the micropipette. As shown in Fig. 2(a), the overall system fits easily in a biological safety cabinet.

Even though a more detailed description of the emulsification dynamics is proposed in Sec. IIC, we now report general observations on the method. For a given microsystem design, and thus hydraulic resistances, the frequency of the droplet formation can be tuned by changing the strength of negative pressure which is here linked to the sampled volume. ${ }^{5}$ Accordingly, the total amount of emulsion is also dependent on the tip's volume. As droplet formation depends on negative pressure generated by micropipette's mechanism, the droplet generation process can be stopped simply by detaching the micropipette from the chip's outlet. This allows to easily collect a portion of the emulsion where droplets have identical size as discussed in Sec. IIC. On the other hand, it is also possible to emulsify all the phase to be dispersed as well as to collect the
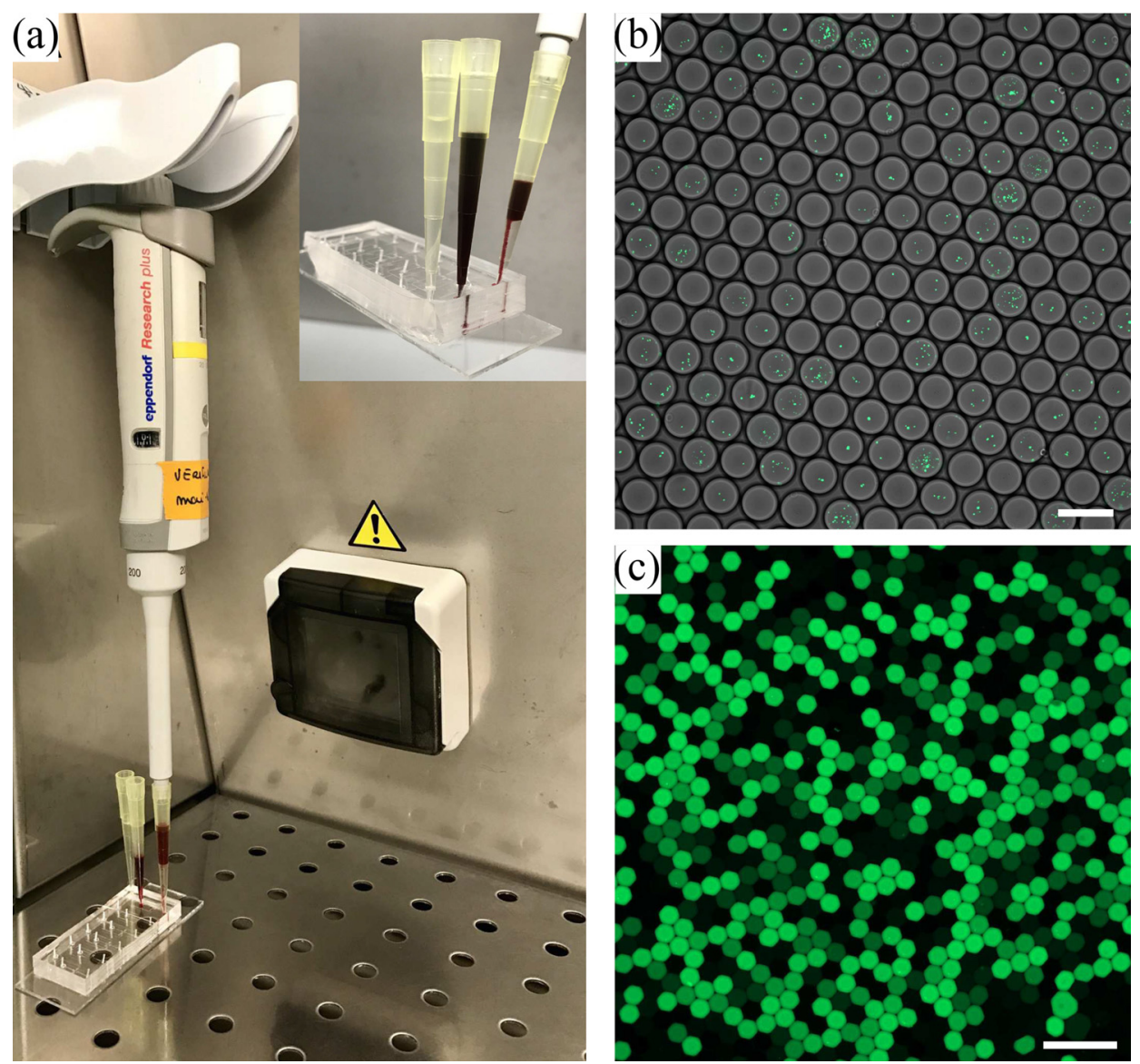

FIG. 2. (a) Snapshots of the micropipette based droplet generation in action in biological safety cabinet. (b) Example of cell (E. coli) encapsulation in droplets produced by the micropipette technique. Scale bar is $200 \mu \mathrm{m}$. (c) The droplet composition can be varied during the emulsification process by using a Taylor dispersion mechanism (see the text for details). Here, quantum dots are encapsulated at an initial concentration of $0.16 \mu \mathrm{M}$ and are revealed by epifluorecence microscopy. 
overall emulsion. The present strategy does not introduce any dead volumes and it is thus well suited to applications where reagents amount matters. Also, in that case, the size distribution is still fairly good, since the corresponding coefficient of variation is less than $8 \%$ when all the aqueous phase is sampled. As discussed later on, the size heterogeneity depends on the emulsified volume for a given micropipette setting. The size homogeneity is indeed very good when a few thousands of droplets are collected as shown in Fig. 2(b). When using the same chip design several times with the identical operating conditions (sampled volume, liquid phases), the relative variation of the mean drop size evaluated on 4 experiments is less than $4 \%$. The reproducibility between batches of emulsion is thus also good, mainly thanks to the robustness of the microfluidic-assisted emulsification and micropipettes.

Finally, it is also possible to change the phase to be dispersed by first unplugging the micropipette and then replacing the tip containing the aqueous phase to another one. After detaching the micropipette, the motion of the plunger should be prevented with the thumb. Since the tip is now filled with water droplets and oil, the next sample volume is lower than the first one. The plunger can be eventually pressed again, after all droplets have creamed, in order to remove air if any or to increase the sampled volume by removing part of the oil. During that operation, since the microchannel where the aqueous phase flows is already filled with the first solution, which may contain solutes, particles, or cells, the suction of the second solution will dilute the first one thanks to a Taylor dispersion mechanism ${ }^{15}$ and vice versa. Indeed, the velocity profile developed in the microchannel leads to an inhomogeneous transit time of solutes, particles, or cells that result in a gradient of concentration. This phenomenon has been exploited to create fine tuned gradients of chemical compounds in droplets for establishing a high resolution dose-response method. ${ }^{16}$ Here, as a proof of concept, quantum dots were encapsulated by following this procedure. The corresponding emulsion is shown in Fig. 2(c). In addition, discretized droplet compositions can be obtained by a parallelization of microfluidic droplet generators, as previously demonstrated with the negative pressure driven flow. ${ }^{5}$

It is also straightforward to connect micropipette tips to any stiff tubes made of polymer, like teflon or PEEK, or metallic, with the help of a softer tube, made in tygon or silicon, for example. In that way, the present methodology based on micropipette can be used with various microsystems other than PDMS ones, like made in glass or with other polymers, for which dedicated connectors for tubing already exists. As a proof of concept, we used commercially available chips made of plastic and obtained a similar emulsification regime with a coefficient of variation of the droplet size around 5\%.

\section{Dynamics of droplet generation}

In order to assess the robustness of the micropipette-based droplet generator, we have recorded images of droplets during the whole emulsification period. This enabled us to obtain a more precise description of the droplet formation dynamics. The droplet's length $L_{d}$ is reported in Fig. 3(a) as a function of time for various suction volumes dV of the micropipette. We note that the droplet's size is initially constant and then slightly decreases over time. The size reduction occurs sooner for a lower suction volume. For all the experiments, the droplet length is larger than the width $w$ of the microchannel, which is here equal to $65 \mu \mathrm{m}$. This is a signature of the squeezing regime of emulsification where the inner phase stream periodically fills the exit channel at the cross junction and breaks due to the flow of the continuous phase. ${ }^{17,18}$ The maximal capillary number, $C a=u_{c} \eta_{c} / \gamma$, where $\eta_{c}$ is the viscosity of the continuous phase, $u_{c}$ is the characteristic velocity of the continuous phase, and $\gamma$ is the interfacial tension, is $7 \times 10^{-3}$, which indeed justifies that droplet formation is in the squeezing regime. ${ }^{19}$ For this regime, the droplet volume $V_{d}$ is given by the sum of an initial volume $V_{0}$ needed to fill, and thus to block the exit channel at the cross junction and the volume of the inner phase injected during the time needed for the outer phase to break the liquid finger. The droplet volume is simply $V_{d}=V_{0}+\alpha r_{q} w^{2} h$, where $r_{q}$ is the ratio of the inner flow rate $q_{i}$ and the outer one $q_{o}, h$ is the channel height equal to $85 \mu \mathrm{m}$, and $\alpha$ is a constant close to 1 . Since the droplet's length is larger than the channel's width, which is larger than the channel's height, the droplet is 
(a)

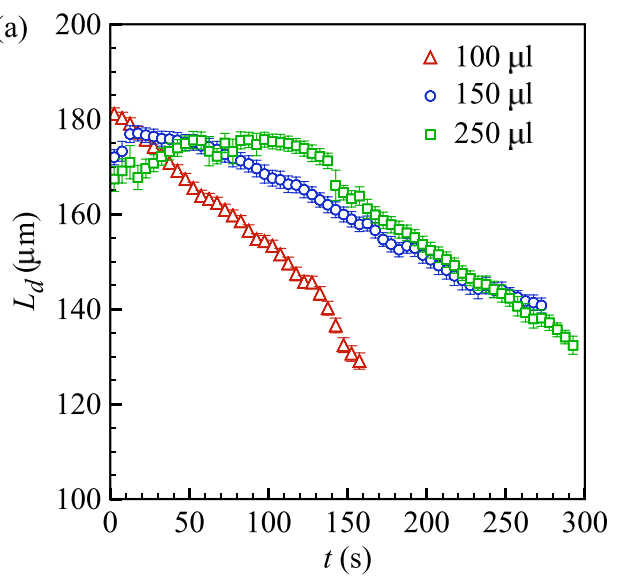

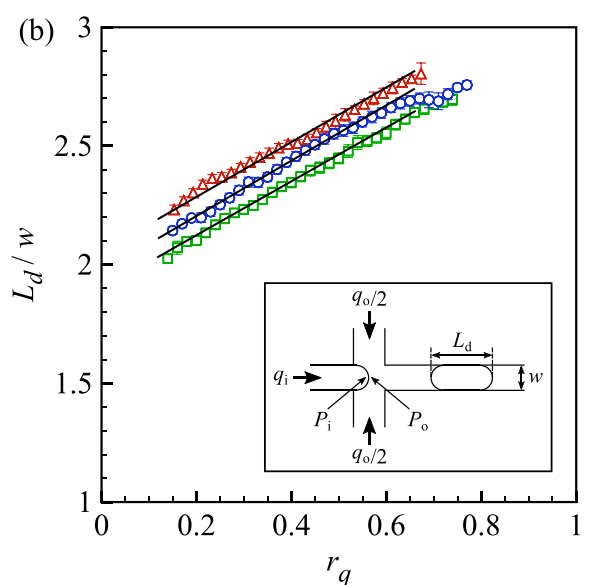

FIG. 3. (a) Time evolution of the droplet's length for various suction volumes dV. (b) Corresponding correlation between the droplet's length normalized by the channel's width as a function of the ratio between the flow rate of the inner phase and the outer one. Inset: Schematic of the cross-junction where droplets are formed along with flow and geometrical parameters.

flattened. The droplet's volume is then approximated by $V_{d}=\left(L_{d}-w\right) w h+\pi / 4 w^{2} h$. Finally, the droplet's length is simply given by

$$
L_{d} / w=\beta+\alpha r_{q},
$$

where $\beta=V_{0} /\left(w^{2} h\right)+1-\pi / 4$ is a constant. The droplet's length normalized by the channel's width is plotted in Fig. 3(b) as a function of the flow rate ratio, estimated by the volume ratio of the two liquid phases. The values of $\alpha$ and $\beta$, given in Table I, are obtained from a linear fit of the data represented by the continuous lines in Fig. 3(b).

We now aim at rationalizing the time evolution of the droplet's size reported in Fig. 3(a) with the help of a simple model. A schematic of a microsystem with a flow focusing geometry for producing droplet is reported in Fig. 4(a). It is characterized by three hydrodynamic resistances representing parts of the whole device: the circuits of the inner and outer phases up to the cross-junction, $R_{i}$ and $R_{o}$, respectively; and the outlet channel where the emulsion droplets flow, $R_{e}$. The liquid height in the micropipette's tip connected to the inlets being around $10 \mathrm{~mm}$, the corresponding hydrostatic pressure is of the order of $100 \mathrm{~Pa}$ and thus neglected in the following. The pressure at both inlets is therefore equal to the atmospheric pressure $P_{\text {atm }}$.

Let us denote $P_{i}, P_{o}$, and $P_{e}$ as the pressures at the cross junction in the inner phase and the outer phase and in the collecting pipette's tip, respectively. The initial pressure $P_{e}(0)$ is linked to the initial suction volume $d V$ of the micropipette via the ideal gas law, $P_{a t m}(V-d V)$ $=P_{e}(0) V$, where $V$ is the volume of the micropipette's tip connected to the micropipette at rest. It is equal to $340 \mu \mathrm{l}$ for our $200 \mu \mathrm{l}$ micropipette tips. The pressure $P_{e}$ is then relaxing towards $P_{a t m}$, while the emulsion is collected in the micropipette at a flow rate $q_{e}$. The time evolution of the pressure inside the tip attached to the micropipette is

TABLE I. Values of some of the parameters used in the model for various aspirated volumes $d V$ : coefficients $\alpha$ and $\beta$ from Eq. (1) obtained by fitting experimental data of droplet's length [Fig. 3(b)], hydraulic resistance $R_{e}$ adjusted such that the time when the droplet production ends, matches with the experimental one [Fig. 3(a)]. The corresponding maximal volume of the inner phase that can be emulsified $V_{m}$ is also reported.

\begin{tabular}{lcccc}
\hline \hline$d V(\mu \mathrm{l})$ & $\alpha$ & $\beta$ & $\operatorname{Re}\left(10^{12} \mathrm{~Pa} \mathrm{~s} / \mathrm{m}^{3}\right)$ & $V_{m}(\mu \mathrm{l})$ \\
\hline 100 & 2.1 & 1.0 & 7.5 & 34.5 \\
150 & 2.0 & 1.1 & 22.5 & 48 \\
250 & 1.9 & 1.1 & 26.0 & 90 \\
\hline \hline
\end{tabular}



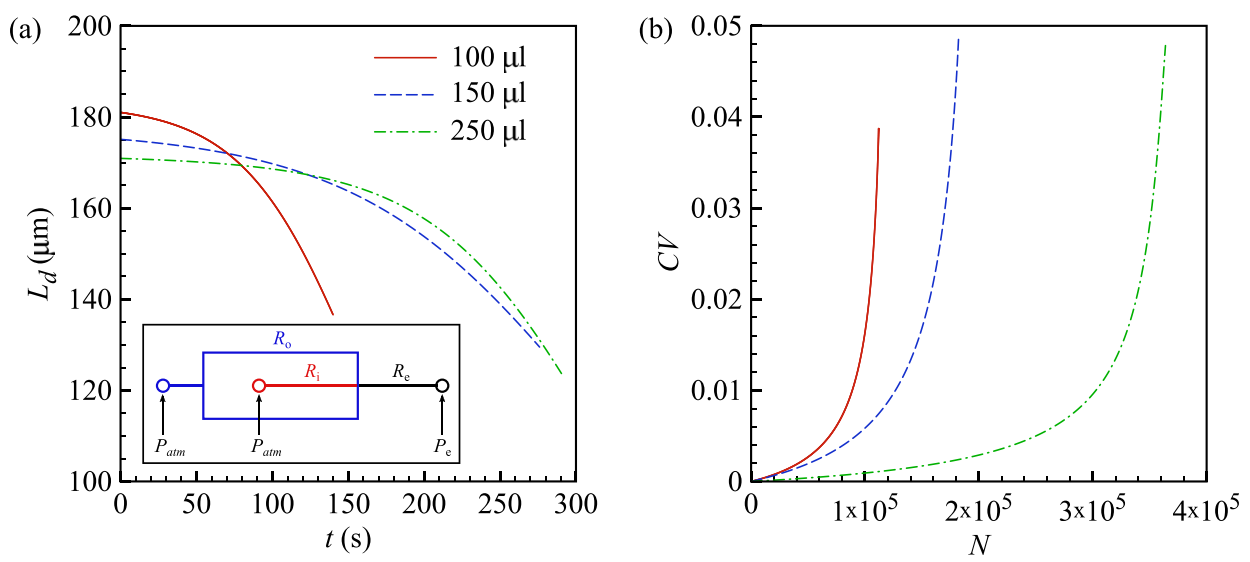

FIG. 4. (a) Model prediction of the time evolution of the droplet's length for the same suction volumes dV reported in Fig. 3. Inset: Schematic of the microsystem introducing the three hydrodynamic resistances considered in the model as well as the pressure condition at the inlets and outlet. (b) Model prediction of the coefficient of variation CV of the drop size as a function of the number of droplets collected in the exit micropipette's tip for the same conditions as in (a).

$$
P_{e}(t)=\frac{P_{e}(0) V}{V-\int_{0}^{t} q_{e}(t) d t} .
$$

The emulsion flow rate $q_{e}$ is the sum of the inner flow rate $q_{i}$ and the outer flow rate $q_{o}$, i.e.,

$$
q_{e}(t)=q_{i}(t)+q_{o}(t) .
$$

The flow rates are linked to the above-mentioned hydraulic resistances and pressures as $q_{i}=\left(P_{a t m}-P_{i}\right) / R_{i}, q_{o}=\left(P_{a t m}-P_{o}\right) / R_{o}$ and $q_{e}=\left(P_{o}-P_{e}\right) / R_{e}$. At the cross junction, there exists a pressure jump at the interface between the inner phase and the outer phase due to the interfacial tension $\gamma$ between the two liquids and the curvature of the interface, namely, the capillary pressure $P_{c}$. In the present emulsification regime, the liquid finger is moving back and forth while being periodically pinched-off. On average, the mean curvature is solely given by the geometry of the microsystem, i.e., its height and width. The capillary pressure is thus approximated to

$$
P_{c}=P_{i}-P_{o}=\gamma\left(\frac{2}{h}+\frac{2}{w}\right) .
$$

By using the expressions of the flow rates and Eq. (3), the flow rate of the outer phase is given by

$$
q_{o}(t)=\frac{P_{a t m}}{R_{o}}\left(\frac{1-p_{e}(t)-p_{c} / r_{i}}{1+1 / r_{i}+1 / r_{o}}\right)
$$

and the inner phase flow rate is

$$
q_{i}(t)=\frac{P_{a t m}}{R_{i}}\left(\frac{1-p_{e}(t)-\left(1+2 / r_{i}+1 / r_{o}\right) p_{c}}{1+1 / r_{i}+1 / r_{o}}\right),
$$

where $r_{o}=R_{o} / R_{e}, r_{i}=R_{i} / R_{e}, p_{c}=P_{c} / P_{a t m}$, and $p_{e}=P_{e} / P_{a t m}$. The flow rate ratio is finally given by

$$
r_{q}(t)=\frac{q_{i}}{q_{o}}=\frac{r_{o}}{r_{i}}\left(1-\left(1+\frac{1}{r_{i}}+\frac{1}{r_{o}}\right) \frac{p_{c}}{1-p_{e}(t)-p_{c} / r_{i}}\right) .
$$


The set of Eqs. (2), (3), and (5)-(7) is then solved numerically by assuming that the hydrodynamic resistance $R_{e}$ of the exit channel is constant. The time evolution of the droplet's length is finally obtained by using Eq. (1). Predictions of the model for various suction volumes are reported in Fig. 4(a) by using the geometrical features of the microsystem, micro pipette's tip and physical properties of liquids used for the experimental data shown in Fig. 3. The hydraulic resistance $R$ of a rectangular channel is ${ }^{20}$

$$
R=\frac{h^{3} w}{12 \eta_{c} L}\left(1-\frac{192}{\pi^{5}} \frac{h}{w} \sum_{n, \text { odd }}^{\infty} \frac{\tanh (n \pi w / 2 h)}{n^{5}}\right),
$$

where $L$ is the channel's length. The estimations of the hydraulic resistances $R_{i}$ and $R_{o}$ from the microsystem's design are $9 \times 10^{12} \mathrm{Pas} / \mathrm{m}^{3}$ and $6.3 \times 10^{12} \mathrm{Pas} / \mathrm{m}^{3}$, respectively. The interfacial tension between the two fluids, measured by the pending drop method, is $9 \mathrm{mN} / \mathrm{m}$. The only unknown parameter is the resistance $R_{e}$ of the exit channel where emulsion droplets were flowing. Its value, reported in Table $\mathrm{I}$, is adjusted for each experiment in order to match with the time at which the droplet production stops, i.e., when $p_{e}^{*}=1-\left(1+2 / r_{i}+1-r_{o}\right) p_{c}$. It is difficult to predict its value as it depends on droplet's velocity, volume fraction of the dispersed phase and channel geometry. ${ }^{21}$ Here, we note that the average value of $R_{e}$ is increasing with the aspirated volume, concomitant to a higher flow rate and thus a higher hydrodynamic resistance introduced by the droplets. ${ }^{22}$ As shown in Fig. 4(a), the dynamics of droplet formation is well accounted for by this simple model. The evolution of the droplet's volume during the emulsification process is linked to the capillary pressure [Eq. (4)] that introduces a difference of the time evolution of the pressure drop between the two feeding reservoirs and the crossjunction. Indeed, such a difference induces a modification of the flow rate ratio that governs droplet's length [Eq. (1)].

Knowing the time evolution of the flow rates, one can estimate the number of droplets formed with time and thus the corresponding time evolution of the droplet size distribution. The coefficient of variation $C V$, defined by the ratio between the standard deviation of the droplet size and its mean value, is reported in Fig. 4(b) as a function of the number of produced droplets in the same operating conditions as in Fig. 4(a). The final size polydispersity of the emulsion is close to $5 \%$, which is consistent with the measured one. This size homogeneity can thus be reduced by stopping the emulsification process, either by using a small volume to be dispersed or by unplugging the micropipette from the microfluidic chip, while having a reasonable amount of droplets. Indeed, by using the largest aspirated volume, a CV of the order of $1 \%$ can be obtained while producing up to $3 \times 10^{5}$ droplets.

The maximal volume $V_{m}$ of the aqueous that is finally emulsified is also indicated in Table I. This emulsified volume is of course linked not only to the initial pressure imposed by the micropipette, i.e., $P_{e}(0)=(1-d V / V) P_{a t m}$, but also on channels' geometry and fluid properties that set the stop pressure, $P_{e}^{*}=P_{a t m}-\left(1+2 R_{e} / R_{i}+R_{e} / R_{o}\right) P_{c}$. Here, the total volume of droplets is about one third of the aspirated volume $d V$.

\section{CONCLUSION}

In this article, we have demonstrated the usefulness of a classical laboratory micropipette for droplet-based microfluidics. Indeed, once coupled to a droplet maker microsystem, such a device acts as an adjustable negative pressure generator for making emulsion droplets. The main advantage over other techniques is the ability to handle small volumes while minimizing dead volumes. In that way, a complete emulsification of precious samples can be achieved. Fortunately, even though small fluid volumes are involved and lead to time dependent flow conditions, this strategy still allows to keep homogeneous drop sizes. The emulsification dynamics is rationalized with a simple model that links liquid properties, channel, and micropipette's tip dimensions to emulsification features, in terms of drop size and size distribution. The regime of drop formation under study corresponds to a squeezing regime where drop size is governed by the flow rate ratio between the two liquids. Therefore, even though the pressure inside the tip 
attached to the micropipette is relaxing towards the atmospheric pressure, leading to a decrease in both flow rates, the drop size is initially constant with time. Then, the average capillary pressure at the interface between the two liquids introduces a time dependent unbalance of the pressure drop between the two feeding reservoirs and the cross-junction. This non stationary pressure difference leads to a non-stationary flow rate ratio and thus a slight decrease in the droplets' size.

The present methodology also allows for several droplet generation scenarios, like a single sample droplet content or with a gradient of composition by successive replacements of the sampled tip. The collection tip is a convenient reservoir to make the link between the droplet generating chip and any other microfluidic devices allowing not only to re-inject stored droplets but also, if needed, for additional steps to be performed on those droplets, such as incubation, irradiation, mixing with other droplets (including dilution with droplets having different compositions). Due to the use of a well-known and ubiquitous device, the micropipette allows for portability and disposability of all elements that were in contact with contaminated material used as aqueous phase (namely tips and droplet generating chips). The pipette-based system presented in this article has thus a clear advantage over any other techniques developed for droplet generation. Its simplicity allows also droplet generation to be performed under almost any conditions, including compact laboratory bench where space is limited, or if necessary a BSL3/ BSL4 microbiological laboratory hood.

\section{SUPPLEMENTARY MATERIAL}

See Movie in the supplementary material for the workflow of the micropipette based droplet generation.

\section{ACKNOWLEDGMENTS}

This work has received the support of Agence National de la Recherche (DYNAMETAFLUID, ANR-14-CE08-0008). We thank our partners, Catherine Rouzeau, Adilya Dagkesamanskaya, Denis Pompon, and Véronique Anton-Leberre from LISBP for discussion. We also thank Guilhem Chenon for his participation to the elaboration of the movie in the supplementary material.

${ }^{1}$ A. B. Theberge, F. Courtois, Y. Schaerli, M. Fischlechner, C. Abell, F. Hollfelder, and W. T. S. Huck, "Microdroplets in microfluidics: An evolving platform for discoveries in chemistry and biology," Angew. Chem. Int. Ed. 49, 5846 (2010).

${ }^{2}$ H. N. Joensson and S. H. Andersson, "Droplet microfluidics - a tool for single-cell analysis," Angew. Chem. Int. Ed. 51, $12176(2012)$

${ }^{3}$ L. B. Pinheiro, V. A. Coleman, C. M. Hindson, J. Herrmann, B. J. Hindson, S. Bhat, and K. R. Emslie, "Evaluation of a droplet digital polymerase chain reaction format for dna copy number quantification," Anal. Chem. 84, 1003 (2012).

${ }^{4}$ H. Becker and C. Gartner, "Polymer microfabrication technologies for microfluidic systems," Anal. Bioanal. Chem. 390, 89 (2008).

${ }^{5}$ A. R. Abate and D. A. Weitz, "Syringe-vacuum microfluidics: A portable technique to create monodisperse emulsions," Biomicrofluidics 5, 014107 (2011).

${ }^{6} \mathrm{C}$. Li, J. Xu, and B. Ma, "A self-powered microfluidic monodispersed droplet generator with capability of multi-sample introduction," Microfluid. Nanofluid. 18, 1067 (2015).

${ }^{7}$ K. Iwai, K. C. Shih, X. Lin, T. A. Brubaker, R. D. Sochol, and L. Lin, "Finger-powered microfluidic systems using multilayer soft lithography and injection molding processes," Lab Chip 14, 3790 (2014).

${ }^{8}$ S. Begolo, D. V. Zhukov, D. A. Selck, L. Li, and R. F. Ismagilov, "The pumping lid: Investigating multi-material 3d printing for equipment-free, programmable generation of positive and negative pressures for microfluidic applications," Lab Chip 14, 4616 (2014).

${ }^{9}$ F. Dutka, A. S. Opalski, and P. Garstecki, "Nano-liter droplet libraries from a pipette: Step emulsificator that stabilizes droplet volume against variation in flow rate," Lab Chip 16, 2044 (2016).

${ }^{10}$ G. T. Vladisavljevic, I. Kobayashi, and M. Nakajima, "Production of uniform droplets using membrane, microchannel and microfluidic emulsification devices," Microfluid. Nanofluid. 13, 151 (2012).

${ }^{11}$ S. L. Anna, N. Bontoux, and H. A. Stone, "Formation of dispersions using 'flow focusing' in microchannels," Appl. Phys. Lett. 82, 364 (2003).

${ }^{12}$ D. C. Duffy, J. C. McDonald, O. J. A. Schueller, and G. M. Whitesides, "Rapid prototyping of microfluidic systems in poly(dimethylsiloxane)," Anal. Chem. 70, 4974 (1998).

${ }^{13}$ L. Boitard, D. Cottinet, C. Kleinschmitt, N. Bremond, J. Baudry, G. Yvert, and J. Bibette, "Monitoring single-cell bioenergetics via the coarsening of emulsion droplets," Proc. Natl. Acad. Sci. U. S. A. 109, 7181 (2012).

${ }^{14}$ A. J. T. Teo, K.-H. H. Li, N.-T. Nguyen, W. Guo, N. Heere, H.-D. Xi, C.-W. Tsao, W. Li, and S. H. Tan, "Negative pressure induced droplet generation in a microfluidic flow-focusing device," Anal. Chem. 89, 4387 (2017). 
${ }^{15}$ G. I. Taylor, "Dispersion of soluble matter in solvent flowing slowly through a tube," Proc. R. Soc. London, Ser. A 219, $186(1953)$

${ }^{16}$ O. J. Miller, A. E. Harrak, T. Mangeat, J.-C. Baret, L. Frenz, B. E. Debs, E. Mayot, M. L. Samuels, E. K. Rooney, P. Dieu et al., "High-resolution dose-response screening using droplet-based microfluidics," Proc. Natl. Acad. Sci. U. S. A. 109, 378 (2012).

${ }^{17}$ P. Garstecki, H. A. Stone, and G. M. Whitesides, "Mechanism for flow-rate controlled breakup in confined geometries: A route to monodisperse emulsions," Phys. Rev. Lett. 94, 164501 (2005).

${ }^{18} \mathrm{P}$. Garstecki, M. J. Fuerstman, H. A. Stone, and G. M. Whitesides, "Formation of droplets and bubbles in a microfluidic tjunction - scaling and mechanism of break-up," Lab Chip 6, 437 (2006).

${ }^{19}$ M. De Menech, P. Garstecki, F. Jousse, and H. Stone, "Transition from squeezing to dripping in a microfluidic t-shaped junction," J. Fluid Mech. 595, 141 (2008).

${ }^{20} \mathrm{P}$. Tabeling, Introduction to Microfluidics (Oxford University Press on Demand, 2005).

${ }^{21}$ C. N. Baroud, F. Gallaire, and R. Dangla, "Dynamics of microfluidic droplets," Lab Chip 10, 2032 (2010).

${ }^{22}$ F. P. Bretherton, "The motion of long bubbles in tubes," J. Fluid Mech. 10, 166 (1961). 